\title{
Zone optimale du soutirage
}

\author{
Optimum zone of drawing of the sediments
}

par B. Remini*

Université de Blida, Algérie

\section{J.-M. Avenard}

ULP de Strasbourg

The technique of sediments drawing by the sluices of draining can reduce the speed of sedimentation in reservoir of dam and consequently increase the time of life. It must be realised rigorously whereas it is not the case in the majority of the Algerian dams. It has then engendred important problems of management, and sometimes the stopping of the exploitation of the dam. The study of the mud concentration in different dams has permitted to observe a critical value for every type of mud, and to determine something like an "optimum of drawing off" where the suspension density permits the drawing off.

\section{SYMBOLES UTILISÉS :}

$C$ : concentration en particules solides $(\mathrm{g} / \mathrm{l})$

$\Psi$ : flux solide $\left(\mathrm{g} / \mathrm{min} \cdot \mathrm{m}^{2}\right)$

$e:$ porosité

$W_{S}:$ vitesse de chute $(\mathrm{cm} / \mathrm{min})$

$n:$ pente de la droite $\operatorname{Ln} W_{S}=f(\operatorname{Ln} e)$

$W_{P}$ : vitesse de chute d'une particule $(\mathrm{cm} / \mathrm{min})$

$\rho_{m}:$ densité de la suspension

$\rho$ : densité de l'eau

$\Delta \rho / \rho_{m}:$ facteur de pression.

\section{PROBLÉMATIQUE}

Le soutirage des sédiments par les vannes de fond peut réduire considérablement l'envasement des retenues en mettant à profit la présence des courants de densité, phénomène favorable à l'utilisation de cette technique. Il est même possible d'affirmer que dans les pays arides et semi-arides, cette utilisation devrait être la règle. Or force est de constater que le soutirage est mal pratiqué en Algérie, malgré la présence des courants de densité dans la majorité, sinon l'ensemble des retenues, ce qui a engendré des pertes considérables d'eau claire dans certains barrages, ou une évolution rapide de l'envasement dans d'autres provoquant ainsi leur abandon comme pour Cheurfas I en 1966, ou encore le blocage de la vanne de fond comme pour Oued el Fodda en 1948.

* La thèse de M. Remini a retenu l'attention des membres du jury du prix Henri Milon.
Cette idée n'est pas nouvelle, et Dessoliers H. avait dès 1908 parfaitement défini ce qu'il fallait faire : « Au lieu de laisser les vases s'accumuler, se tasser, se durcir, puis s'acharner à les extraire, ce qui sera d'autant plus coûteux que l'on aura attendu plus longtemps, il faut après chaque crue, pendant qu'elles sont encore semi-fluides, les évacuer immédiatement par un égout de fond ». Il est à noter qu'à cette époque l'auteur n'a pas trouvé audience. Allant dans le même sens, H. Duquennois (1954) a montré que les soutirages qui ne se font pas dès l'arrivée du courant de densité auront un rendement plus élevé mais que la quantité totale des sédiments sera inférieure à celle du soutirage immédiat. Il a affirmé aussi qu'il n'est pas nécessaire de soutirer des mélanges très denses, au risque de ne pas évacuer le maximum possible de la vase. Le même auteur avait, dès 1949 , estimé que si l'on pratique l'ouverture des vannes de fond lorsque la consolidation est avancée, on évacue de l'argile et de la vase de densité élevée (plus de 1,350) mais en faible quantité ; on arrive rapidement à l'eau claire.

\section{I INFLUENCE DE LA CONCENTRATION EN ÉLÉMENTS FINS SUR LE « FACTEUR DE PRESSION "}

Le facteur de pression est défini comme étant le rapport entre la variation de la densité $\left(\Delta \rho=\rho_{\mathrm{m}}-\rho\right)$ et la densité de la suspension $\left(\Delta \rho / \rho_{\mathrm{m}}=\left(\rho_{\mathrm{m}}-\rho\right) / \rho_{\mathrm{m}}\right)$. Il est caractérisé comme la force motrice du courant de densité et comme paramètre principal du soutirage.

Plus $\Delta \rho / \rho_{\mathrm{m}}$ est élevé, plus l'évacuation des sédiments sera importante et la perte d'eau sera faible, et il ne doit pas 
dépasser une valeur limite de la concentration qui dépend de la nature et de la granulométrie de la vase.

Selon H. Duquennois (1944), si les vases restent accumulées pendant de longs mois sous l'eau, leur densité augmente en tendant vers une limite. Leur viscosité augmente très sensiblement et bien qu'il s'agisse encore de fluide, la viscosité est telle que l'écoulement ne peut se faire qu'avec de très fortes pertes de charge ». Cette hypothèse a été confirmée par Drouhin et al. (1951).

Nous avons étudié l'évolution du "facteur de pression " en fonction de la concentration des sédiments sur des échantillons de vase évacuée par les pertuis de vidange des barrages de Ghrib, Ooued el Fodda, Zardezas et Foum el Gherza. Les résultats obtenus sont représentés sur la figure 1 ( $\left.\begin{array}{lll}a & a & d\end{array}\right):$ on constate que le «facteur de pression " $\Delta \rho / \rho_{\mathrm{m}}$ évolue linéairement dans une première phase, à partir d'une valeur critique de la concentration, il y a ralentissement de ce facteur, la fonction prenant une forme parabolique.

Les valeurs critiques de concentrations (ou densités) sont consignées dans le tableau 1 .

La même étude a été faite sur des données de concentration enregistrées au cours de différentes opérations de soutirage dans les barrages de Ghrib, Oued el Fodda, Erraguene et Ighil Emda. Les mêmes interprétations peuvent être faites et les résultats obtenus sont représentés sur la figure 2 ( $a$ à d). Les valeurs critiques de concentration (ou densité) sont données sur le tableau 2.

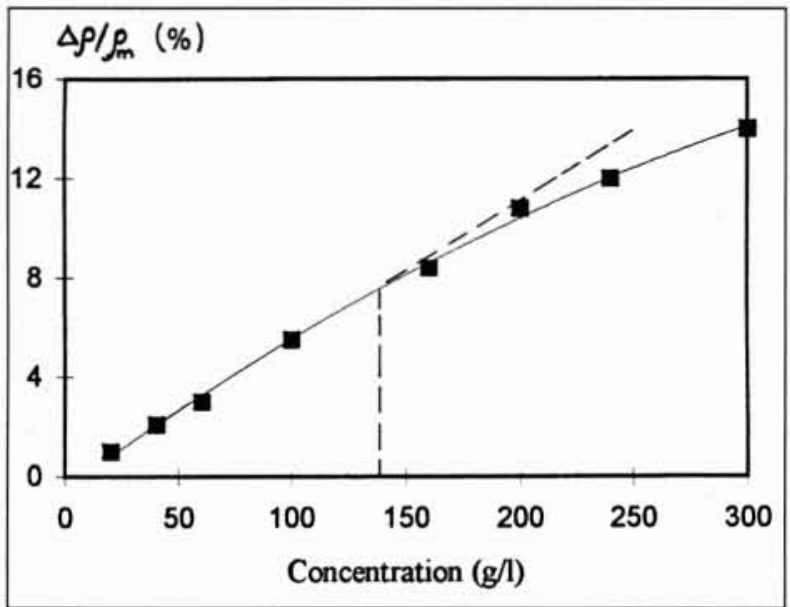

1a. Vase de Oued el Fodda.

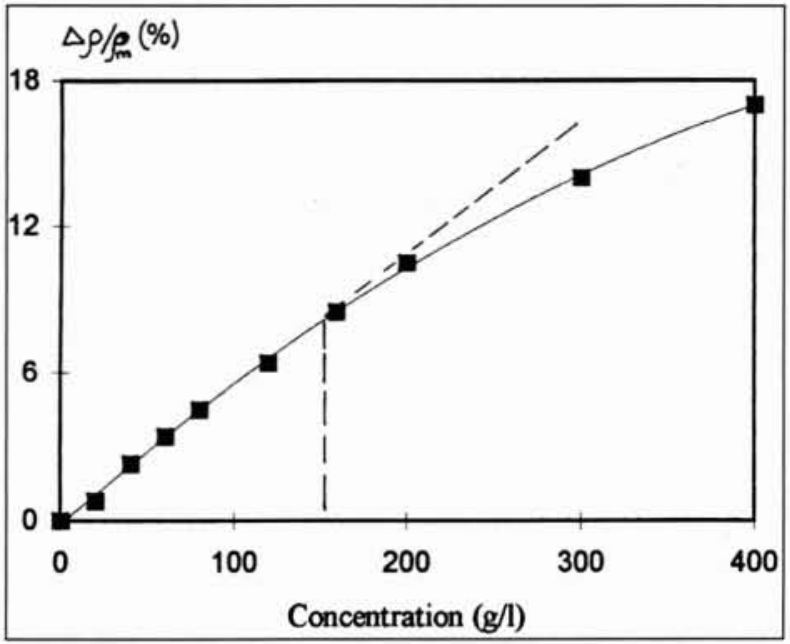

1c. Vase de Zardezas.
Tableau 1. - Valeurs critiques de la concentration de la vase.

\begin{tabular}{|c|c|c|}
\hline \multirow{2}{*}{$\begin{array}{c}\text { Vases } \\
\text { des } \\
\text { barrages }\end{array}$} & \multicolumn{2}{|c|}{$\begin{array}{c}\text { Valeurs critiques } \\
\text { de la concentration }\end{array}$} \\
\cline { 2 - 3 } & Densité $\left(\rho_{\mathbf{m}}\right)$ & Concentration $(\mathbf{g} / \mathbf{l})$ \\
\hline Ghrib & 1,096 & 140 \\
Oued El Fodda & 1,083 & 137 \\
Foum El Gherza & 1,140 & 200 \\
Zardezas & 1,087 & 156 \\
\hline
\end{tabular}

Tableau 2. - Valeurs critiques de la concentration de la vase (enregistrées au cours de différentes opérations de soutirage).

\begin{tabular}{|c|c|c|}
\hline \multirow{2}{*}{$\begin{array}{c}\text { Vases } \\
\text { des } \\
\text { barrages }\end{array}$} & \multicolumn{2}{|c|}{$\begin{array}{c}\text { Valeur critique } \\
\text { de concentration }\end{array}$} \\
\cline { 2 - 3 } & Densité $\left(\rho_{\mathbf{m}}\right)$ & Concentration (\%) \\
\hline Ghrib & 1,096 & $14,7 \%$ \\
Oued El Fodda & 1,082 & $14,0 \%$ \\
Ighil Emda & 1,059 & $10,5 \%$ \\
Erraguene & 1,070 & $11,5 \%$ \\
\hline
\end{tabular}

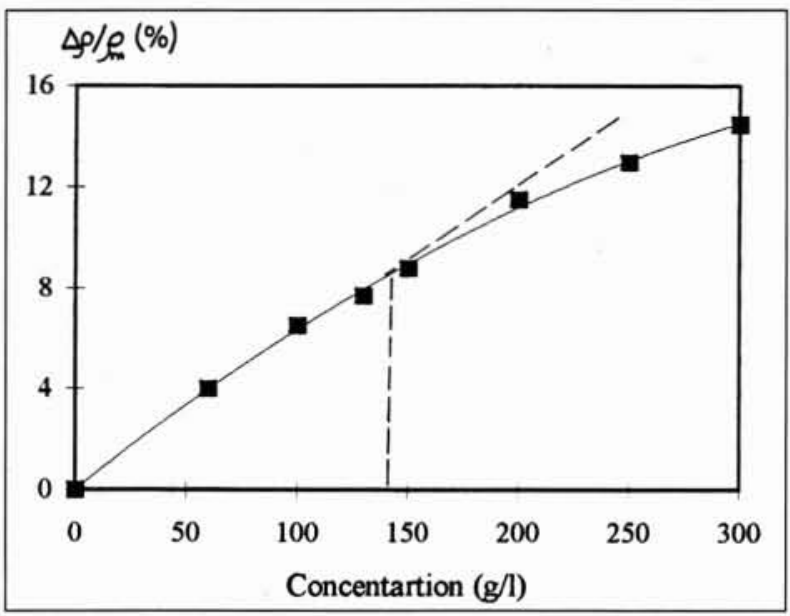

1b. Vase de Ghrib.

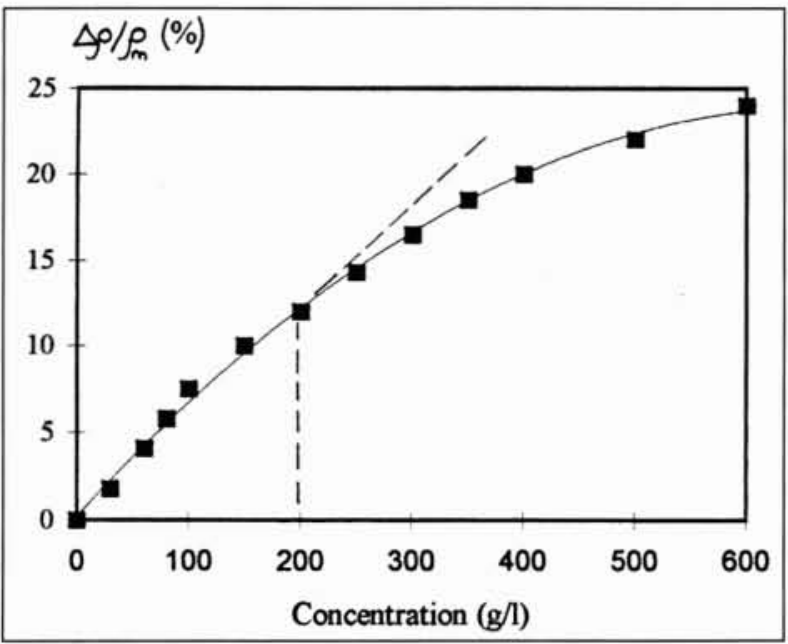

1d. Vase dez Foum el Gherza.

1. Evolution du «facteur de pression » en fonction de la concentration. 


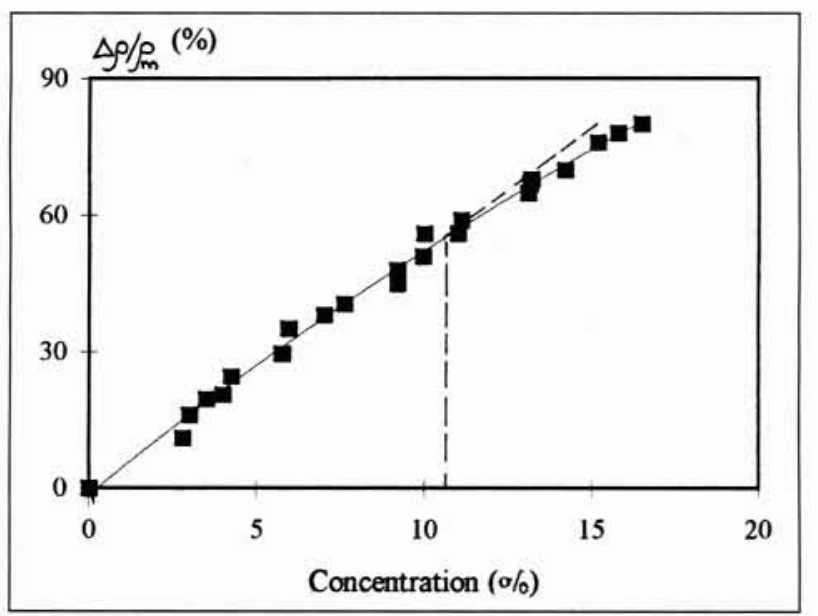

2a. Vase d'Ighil Emda.

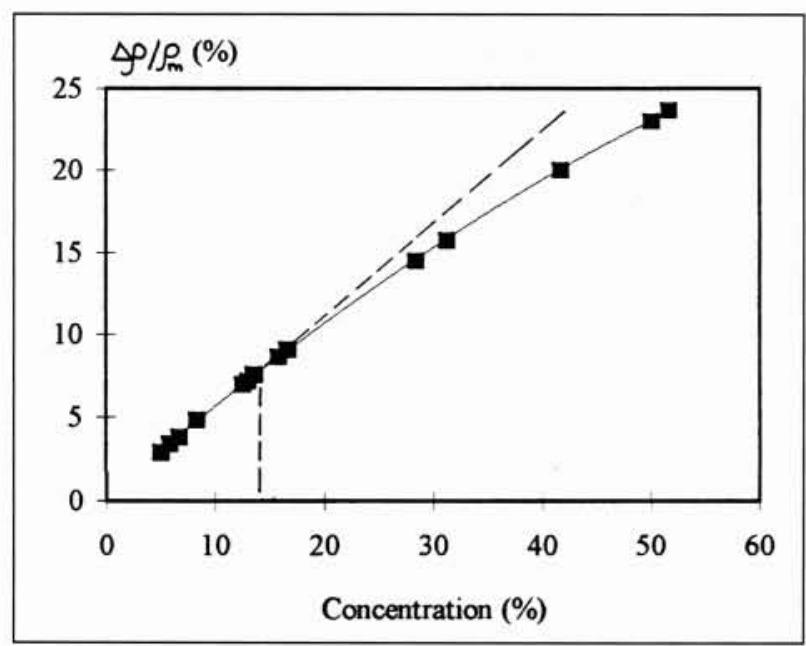

2c. Vase d'Oued el Fodda.

2. Evolution du «facteur de pression » en fonction de la concentration, d'après les données des opérations de soutirage.

En conclusion, nous pouvons dire que la valeur critique de la concentration correspondant à l'apparition de la rigidité initiale est atteinte au changement rhéologique de la suspension : ceci veut dire que la suspension passe d'un liquide Newtonien à un liquide non Newtonien.

\section{FLUX SOLIDE DANS UNE RETENUE}

Dans un milieu solide, la décantation est caractérisée par un flux solide (débit massique par unité de surface) symbolisé par la lettre $\Psi$ et qui est obtenu par la relation :

$$
\Psi=C . W_{s}
$$

Pour différentes valeurs de la concentration, on mesure la vitesse de chute des sédiments. Les résultats obtenus permettent d'établir l'allure de la fonction $L n W s=f($ Lne $)$ (fig. 3 (a à d)) qui est une droite d'équation :

$$
\begin{array}{cc}
\operatorname{Ln} W_{S}=n \operatorname{Ln}(e)+\operatorname{Ln} W_{P}, \\
\text { d'où : } & W s / W p=e^{n}
\end{array}
$$

C'est-à-dire: $\Psi=C . W_{p} e^{n}$. Le flux dépend uniquement la porosité « $e$ ».

Le flux maximum est obtenu pour une valeur de « $e$ » comprise entre 0 et 1 . Il est déterminé par :

$$
d \Psi / d e=W p\left[n e^{n}(1-e)+e^{n}(-1)\right]=0
$$

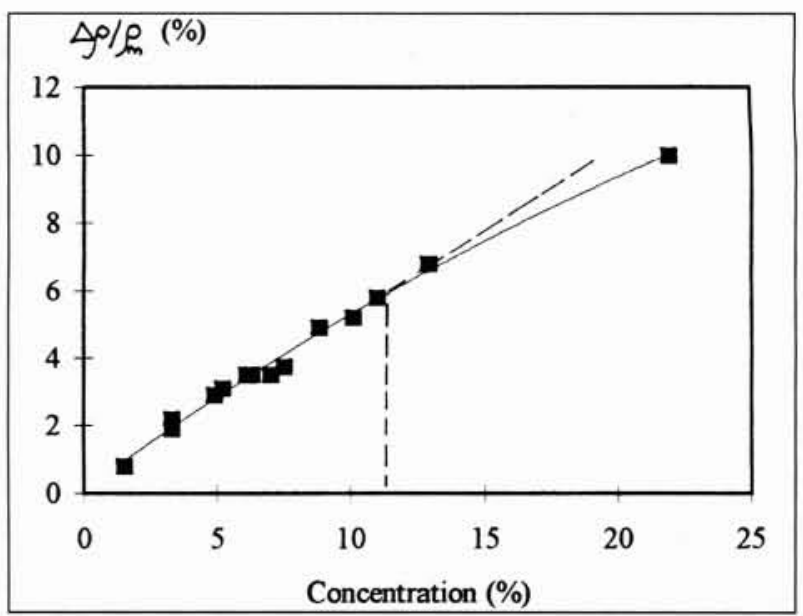

2b. Vase Erraguene.

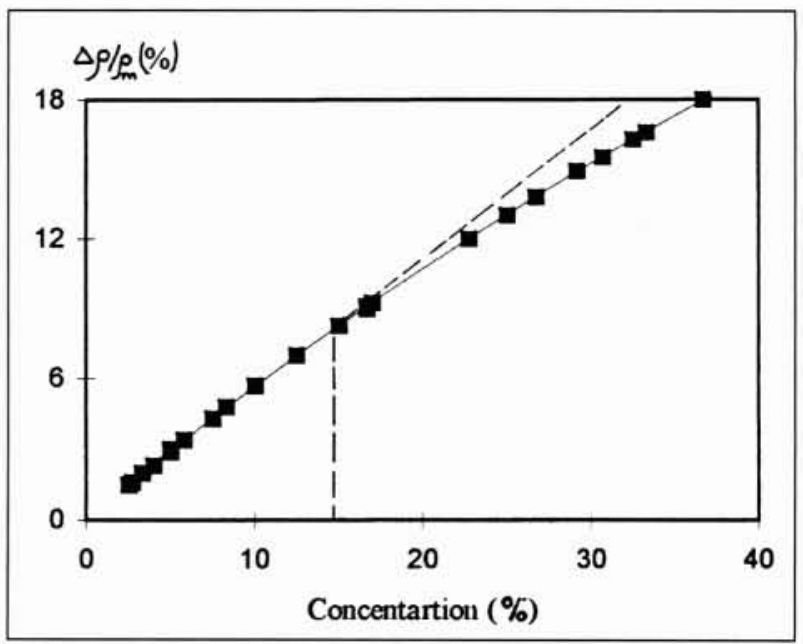

2d. Vase de Ghrib.

d'où :

$$
\mathbf{e}=\mathbf{n} /(\mathbf{1}+\mathbf{n})
$$

De la même manière, nous pouvons déterminer la valeur de l'indice de porosité « $e$ » correspondant au point d'inflexion (point critique) de la courbe $Y=f(e)$ :

$$
\begin{aligned}
& d^{2} Y / d e^{2}=W p\left[n(n-1) e^{n-1}-n(n-1) n e^{n-2}\right]=0 \\
& \text { où : } \quad e=(n-1) /(n+1)
\end{aligned}
$$$$
\text { d'où : }
$$

Nous avons étudié l'influence de la concentration en éléments fins sur le flux solide pour des vases soutirées au niveau des barrages de Ghrib, Oued el Fodda, Zardezas, Ighil Emda, Foum el Gherza et Hamiz. Nous constatons, d'après les résultats consignés sur la figure 4 ( $a$ à $f$ ), que le flux solide suit une loi polynomiale du troisième degré en fonction de la concentration (densités) en sédiments dans la suspension, présentant ainsi un maximum et un point d'inflexion.

La densité (concentration) de la mixture calculée par la relation de l'indice de porosité $e=n /(1+n)=(1-c)$ correspondant exactement à celle du flux solide maximum, ce qui veut dire qu'en ce point, la décantation des particules fines est importante et qu'il y aura même risque de blocage des vannes de fond, en l'absence de soutirage.

Par contre la densité (ou la concentration) de la mixture calculée par la relation de l'indice de porosité $e=(n-1) /(n$ $+1)=1-c$ correspond à celle du point d'inflexion flux solide. Les valeurs obtenues pour les vases des six retenues étudiées sont données dans le tableau 3. 


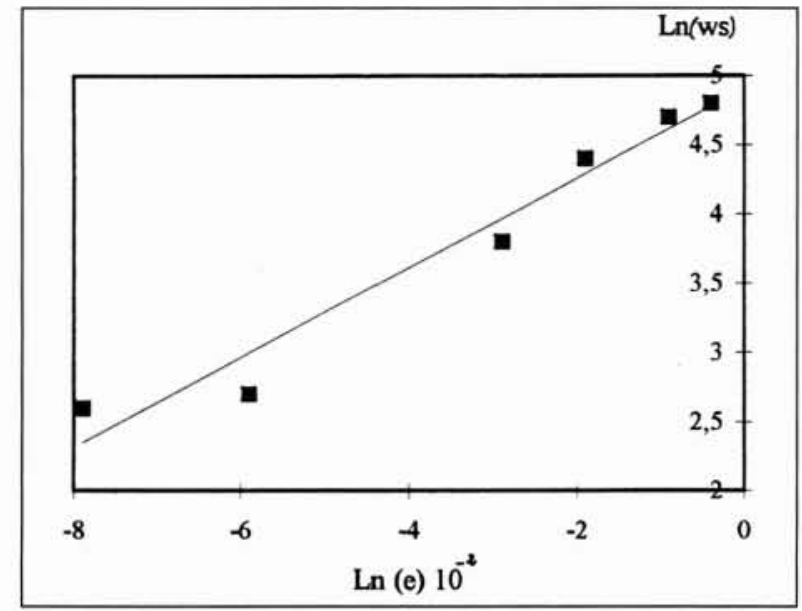

3a. Oued el Fodda.

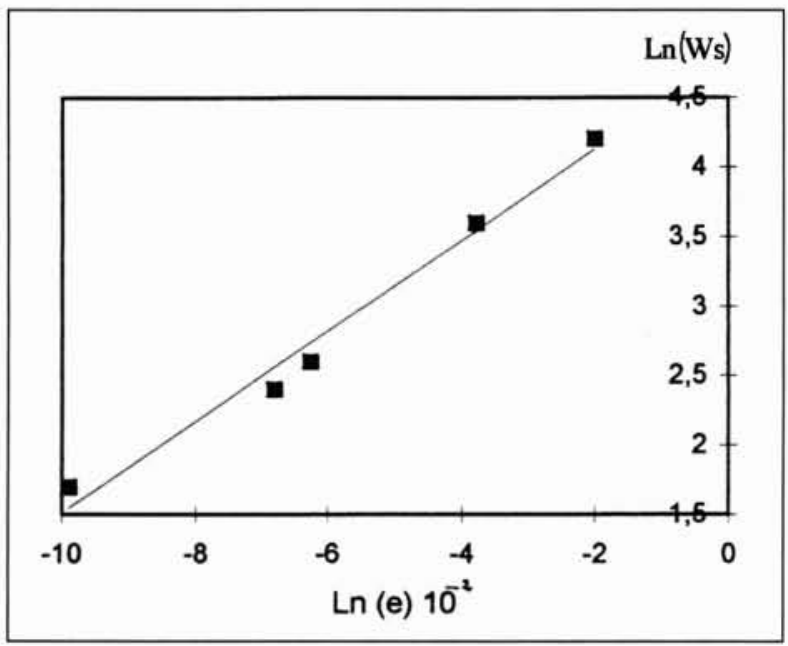

3c. Zardezas.

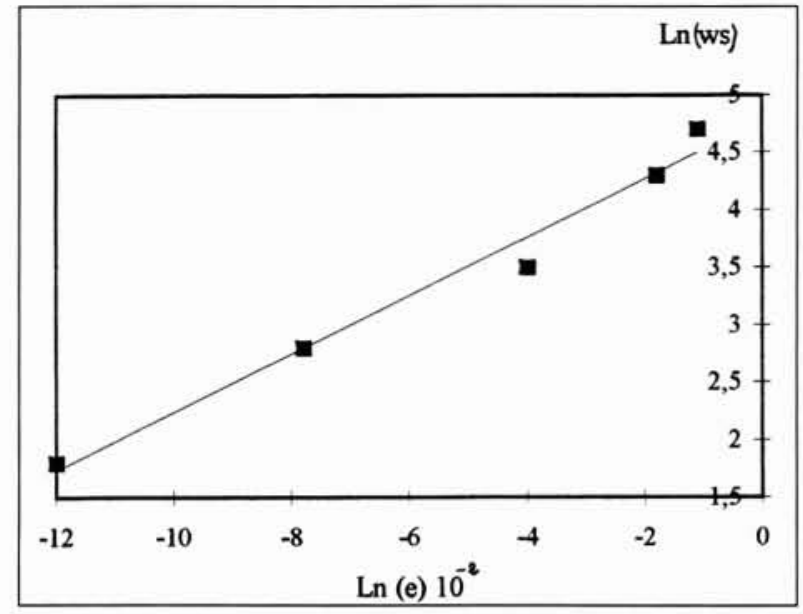

3b. Foum el Gherza.

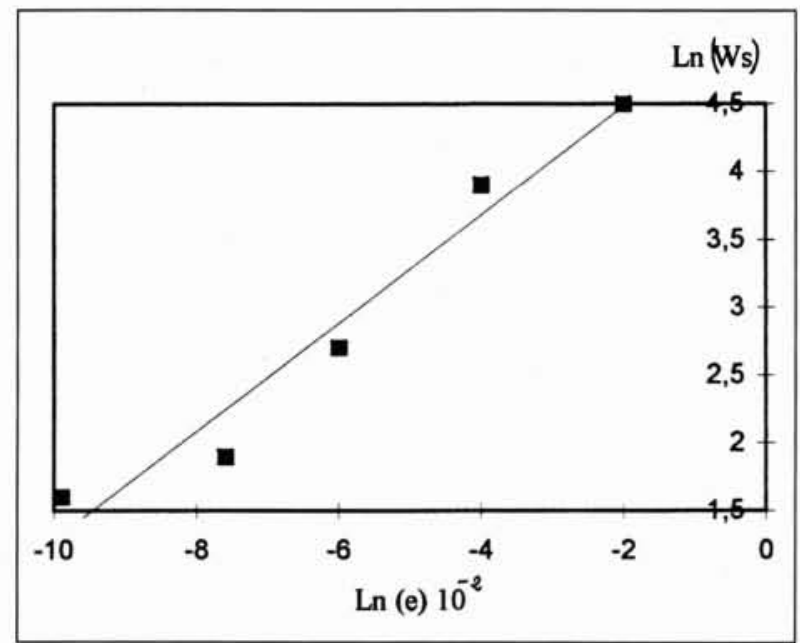

3d. Ghrib.

3. Détermination de la pente « $n »$.

En comparant les valeurs des densités du tableau 3 avec celles correspondant au changement de la fonction du « facteur de pression $"\left(\Delta \rho / \rho_{m}\right)$ données dans les tableaux 1 et 2 , nous remarquons qu'elles sont identiques. Nous pouvons ainsi admettre que le point d'inflexion de la fonction flux solide correspond exactement au point critique d'apparition de la rigidité initiale.

Signalons enfin que H. Duquennois, dès 1954 affirmait que la vase de la retenue d'Ighil Emda ne se comporte plus

Tableau 3. - Concentrations correspondant au valeurs critiques du flux solide.

\begin{tabular}{|c|c|c|}
\hline $\begin{array}{c}\text { Vases } \\
\text { des barrages }\end{array}$ & $\begin{array}{c}\text { Densité } \\
\text { (ou concen- } \\
\text { tration) du flux } \\
\text { maximum }\end{array}$ & $\begin{array}{c}\text { Densité (ou } \\
\text { concentration) } \\
\text { du point } \\
\text { d'inflexion }\end{array}$ \\
\hline $\begin{array}{c}\text { Foum El Gherza } \\
\text { Ghrib } \\
\text { Oued El Fodda } \\
\text { Zardezas } \\
\text { Ighil Emda } \\
\text { Hamiz }\end{array}$ & $\begin{aligned} \mathrm{C} & =100 \mathrm{~g} / \mathrm{l} \\
\mathrm{C} & =75 \mathrm{~g} / \mathrm{l} \\
\mathrm{C} & =67 \mathrm{~g} / \mathrm{l} \\
\mathrm{C} & =78,7 \mathrm{~g} / \mathrm{l} \\
\rho_{m} & =1,030 \\
\mathrm{C} & =110 \mathrm{~g} / 1\end{aligned}$ & $\begin{aligned} C & =200 \mathrm{~g} / / \\
C & =145 \mathrm{~g} / 1 \\
C & =137 \mathrm{~g} / 1 \\
C & =156 \mathrm{~g} / 1 \\
\rho_{m} & =1,058 \\
C & =213 \mathrm{~g} / 1\end{aligned}$ \\
\hline
\end{tabular}

comme un liquide Newtonien si sa densité dépasse la valeur de 1,060. Nos résultats vont ainsi dans le même sens, puisque la valeur critique du début de changement rhéologique du liquide est pour nous de 1,059.

Allant dans le même sens, C. Migniot. et J. Valembois ont étudié l'influence de la concentration en éléments fins sur la rigidité de la vase de Hamiz. La valeur de la concentration d'apparition de la rigidité $(240 \mathrm{~g} / \mathrm{l})$ se rapproche de la nôtre (213 g /).

\section{CONCLUSION}

De la présente étude, il résulte que :

- dans le cas où le soutirage est exécuté pour des densités (ou concentrations) inférieures à la densité du flux maximum, une quantité de matière solide sera évacuée, avec une proportion d'eau claire beaucoup plus élevée ;

- par contre, si le soutirage est exécuté pour des densités supérieures à celle du point d'inflexion, c'est-à-dire que les vases sont restées accumulées pendant un temps assez long sous l'eau, leurs densités augmentent très sensiblement. La suspension n'est plus un liquide newtonien, mais plutôt plas- 


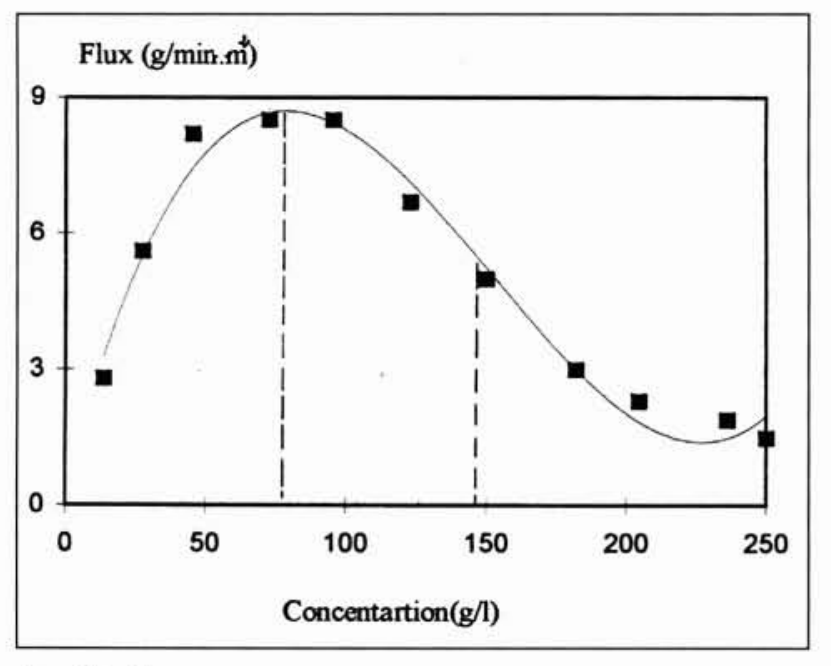

4a. Ghrib.

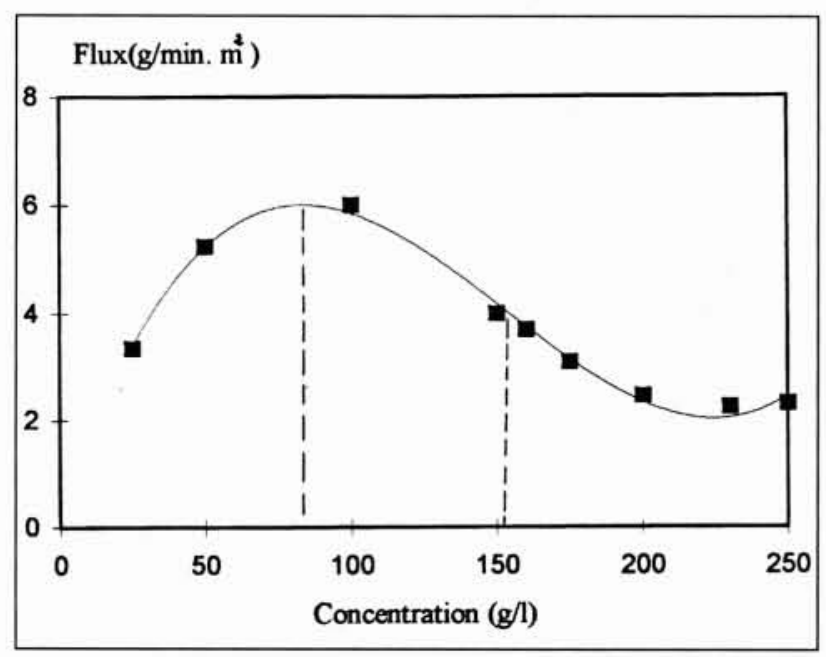

4c. Vase de Zardezas.

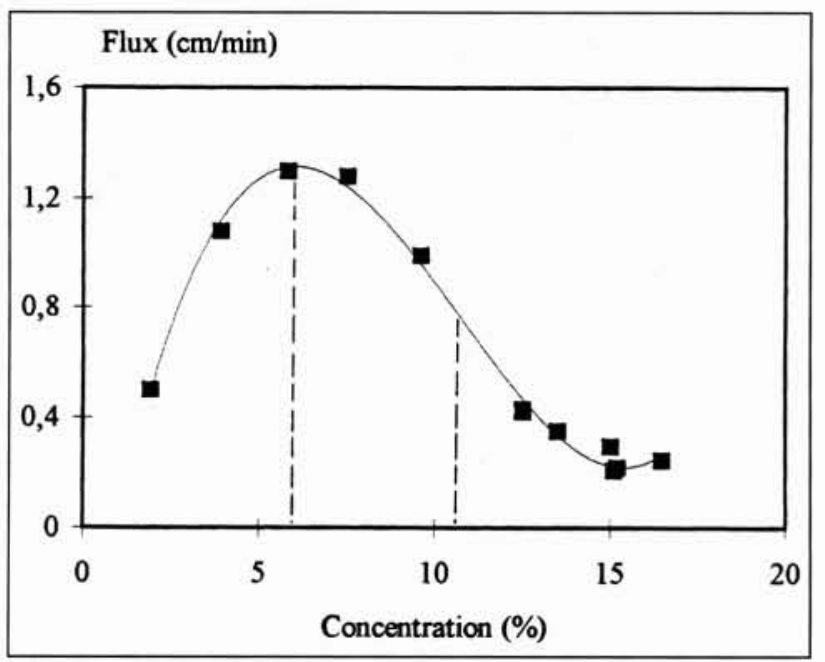

4e. Vase d'Ighil Emda.

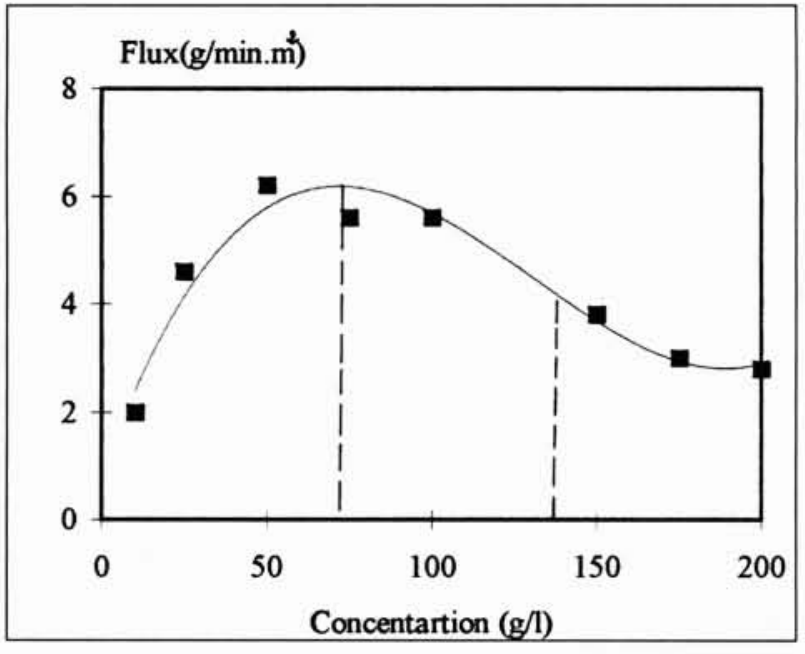

4b. Vase d'Oued el Fodda.

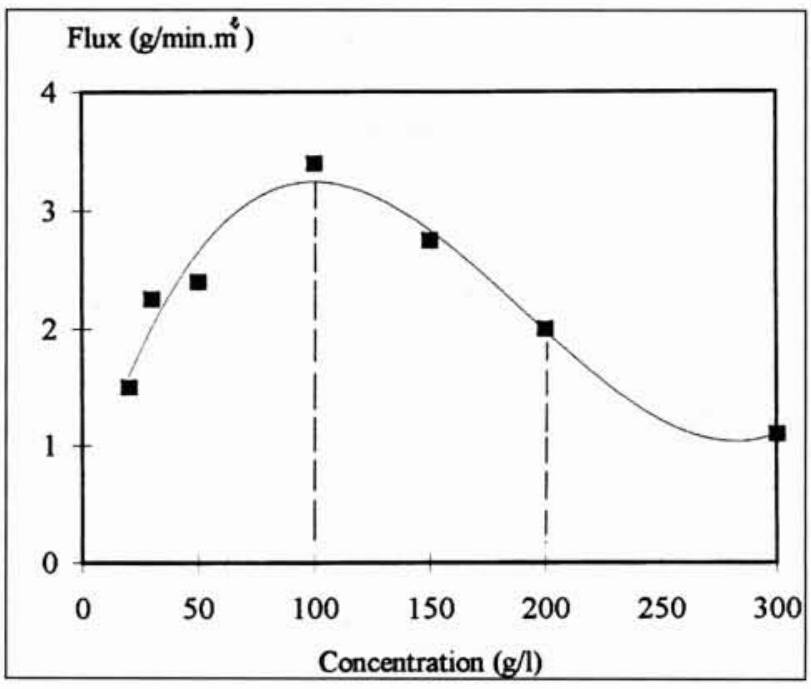

4d. Vase de Foum el Gherza.

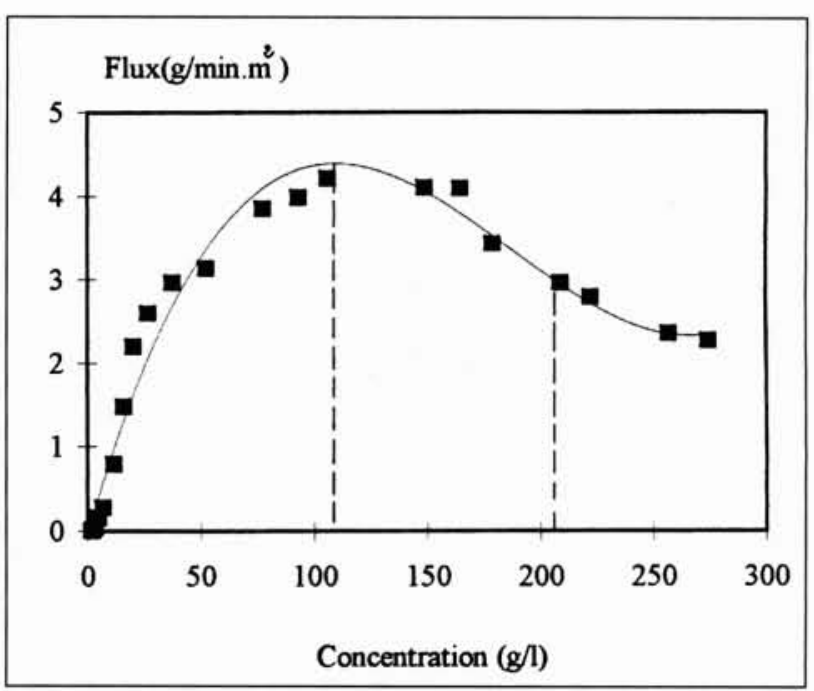

4f. Vase de Hamiz.

4. Variation du flux solide en fonction de la concentration. 
tique. La viscosité augmente aussi de telle sorte que l'écoulement ne peut se faire qu'avec de très fortes pertes de charges; dans ce cas une faible quantité de vase sera évacuée, mais également un faible volume d'eau.

Donc, nous pouvons définir la zone optimale de soutirage comme étant une zone comprise entre la valeur de la densité du flux solide maximum et celle du point d'inflexion du flux solide.

S'il est ainsi possible d'affirmer que la technique du soutirage des sédiments dans les pays arides et semi-arides est une nécessité, elle doit être utilisée efficacement. En effet, pour avoir un bon rendement au niveau du soutirage, en évacuant le maximum de vase et un minimum d'eau claire, nous avons montré qu'il existe une zone optimale de soutirage (Z.O.S.), c'est-à-dire que l'évacuation des sédiments doit se faire avec des densités comprises dans cette zone.

\section{RÉFÉRENCES}

[1] Dessoliers H. in Brown C.B. (1944). - "The control of reservoir sitting". Publication n 521, U.S. Département of agriculture.

[2] DuquenNoIs H. (1956), - Lutte contre la sédimentation des barrages réservoirs. Barrage d'Ighil Emda, Gaz et Electricité d’Algérie. C.R. n 3, Année 1955-1956.

[3] Duquennois H. (1949). - Le débit solide et les barrages réservoirs - électricité et gaz d'Algérie. Rapport, mars, 25 pages.

[4] Duquennois H. (1944). - Note sur les enseignements à tirer pour lutter contre l'envasement des grands barrages réservoirs. Note du 30 octobre. Service d'électricité, 2 pages.

[5] Remeneras G. et Braudeau G. (1951). - Quelques observations sur 1'alluvionnement dans les réservoirs Français. Quatrième congrès des grands barrages. New Delhi, question $n^{\circ} 14$, pp. 197-235.

[6] REMinı B. (1997). - Envasement des retenues de barrages en Algérie : importance, mécanismes et moyen de lutte par la technique du soutirage. Doctorat d'état, Ecole Nationale Polytechnique d'Alger, mars, 345 pages.

[7] Reminı B. et al. (1995). - Envasement du barrage d'Ighil Emda (Algérie). Revue la Houille Blanche n $2 / 3$, pp. 23-28.

[8] Reminı B. et al. (1996). - Barrage d'Igil Emda. Les soutirages des courants de densité. Revue Vecteur Environnement (Canada), vol. 29, n 4, août, pp. 27-32.

[9] Reminı B. et al. (1997). - La technique du soutirage. Un moyen de lutte contre l'envasement. Revue Sciences Techniques et Méthodes n³, mars, pp. 69-76. 